\title{
The impact of allergic rhinitis on work productivity
}

\section{Sheryl L Szeinbach ${ }^{a, *}$, Enrique C Seoane-Vazquez ${ }^{\mathrm{a}}$, Andrew Beyer ${ }^{\mathrm{a}}$, P Brock Williams ${ }^{b}$}

a College of Pharmacy, Ohio State University, Columbus, Ohio, USA

b University of Missouri Medical School, Kansas City, Missouri, USA

Received 8th September 2006; accepted 20th December 2006

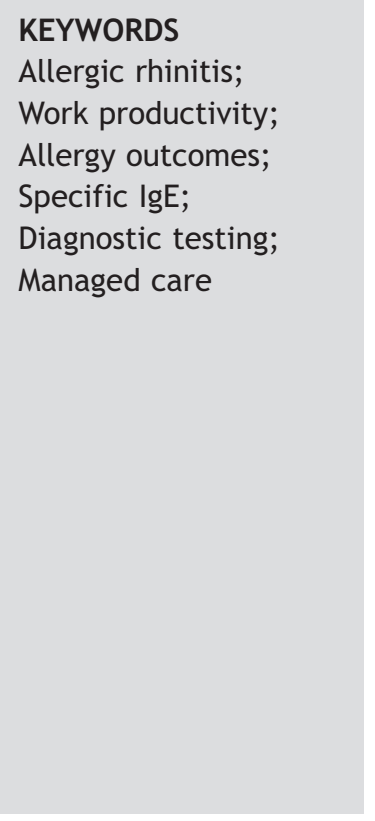

\begin{abstract}
Aim: Patients with allergic rhinitis experience a multitude of symptoms that usually compromise some aspect of lifestyle. However, few data are available that specifically address the impact of allergic rhinitis on work productivity.

Methods: A questionnaire was developed and mailed to 2,065 patients enrolled in a 500,000-member managed care organisation. Patients were identified by diagnostic codes for allergic rhinitis as determined by a retrospective examination of medical and prescription claims records from January 12000 to December 31 2000. Patients were divided into three different care groups according to whether they were managed by family physicians, by allergists, or were self-managed.

Results: Chi-square and analysis of variance tests revealed significant differences among the three care groups $(\mathrm{p}<0.05)$ for years with allergies, symptoms, family history, testing, immunotherapy, test value, and prescribed antihistamine use. Multiple linear regression analysis revealed that sleep, health, certain allergy symptoms and prescribed antihistamines were significantly related to work productivity.

Conclusions: The results of this study revealed that the ability of individuals with allergic rhinitis to engage in productive work is influenced by sleep, health-related quality of life (HRQoL), specific symptoms, and prescribed antihistamine use. ๑) 2007 General Practice Airways Group. All rights reserved.
\end{abstract}

* Corresponding author.

College of Pharmacy, The Ohio State University, 500 W 12th Ave, Columbus, OH 43210, USA

Tel: +1 614-688-4249; fax: +1 614-529-5070

E-mail address: Szeinbach.1@osu.edu

\section{Introduction}

For the 20 to 50 million Americans who experience symptoms associated with allergic rhinitis, their symptoms have a pronounced impact on many aspects of daily living and lifestyle. ${ }^{1,2}$ With current allergy prevalence estimates of $10 \%$ to $40 \%,{ }^{3,4}$ changing environmental conditions support an increasing worldwide prevalence of allergic rhinitis and other conditions such as asthma. ${ }^{5,6}$ In fact, for

1471-4418 @ 2007 General Practice Airways Group. All rights reserved doi:10.3132/pcrj.2007.00015 
young patients with more persistent and severe symptoms of allergic rhinitis, there is a three-fold higher risk for developing asthma later in life. ${ }^{6}$ Although conditions such as asthma and rhinosinusitis are likely to coexist with allergic rhinitis, making it difficult to distinguish by symptoms alone, treatment is contingent on a thorough clinical evaluation and an accurate diagnosis.

Clinical evaluation and history gleaned from patients is usually supported by some type of diagnostic testing - either skin testing or specific IgE (slgE) blood testing. However, the usefulness of skin testing by family physicians is limited because of the apparent expertise needed for interpretation of skin test results, which is not necessarily straightforward. Nonetheless, the slgE test has been acknowledged as an accurate tool, and may be useful to family physicians for identifying specific allergen sensitivities and for determining the need for further investigation and referral. ${ }^{7,8}$ Because this test is highly specific and sensitive for lgE antibodies to most prevalent inhalant allergens, ${ }^{9-12}$ testing provides family physicians with an accurate and easily interpretable baseline assessment of allergic disease. An accurate diagnosis is important to evaluate the extent to which lifestyle, health-related quality of life (HRQoL), and symptoms, are influenced by treatments or other options which could influence work productivity.

Patients with allergic rhinitis usually experience a multitude of symptoms including nasal passage inflammation, ocular changes and throat irritation, and can also experience cognitive effects, mood disturbances, and fatigue. ${ }^{13-15}$ Besides the effect of decreased worker productivity, ${ }^{16,17}$ the impact of allergic rhinitis on functional, physical, and psychological activities significantly decreases patient HRQoL. ${ }^{18,19}$ Although prescribing of antihistamine drugs may alleviate symptoms in some patients, a definitive diagnosis should result in more appropriate clinical management, less frequent medication changes, and improved quality of life. ${ }^{20}$ However, without sufficient data, it is difficult to ascertain the impact that prescribed antihistamines and other aspects of lifestyle have on work productivity.

Therefore, this study uses data from a large managed care organisation to examine the relationship between HRQoL, medication use, symptom severity and sleep, on work productivity. As guidelines for the management of allergic rhinitis continue to evolve, the identification of variables associated with work productivity would provide a useful contribution to the care process in the management of these patients.

\section{Methods}

\section{Sample}

Records for patients enrolled in a 500,000-member Health Alliance Plan (HAP) affiliated with the Henry Ford Health System (HFHS) were examined retrospectively for patients who were suspected of having allergic rhinitis by three members of the health outcomes group. Patient criteria for inclusion were: age over 18 years old as at January 1st 1998; and being continuously enrolled in the HAP during the study time frame from January 1st to December 31st 2000.

Once patients were identified, medical and prescription claims were examined to document and verify test dates, test code descriptions, medication use, and ordering physician. Of these 500,000 enrolled members, there were 2,065 patients with a recent diagnosis of allergic rhinitis (ICD-9-CM 477.0, 477.8, and 477.9 for pollen, other allergens, and unspecified, respectively). The inhalant in vitro allergy panel consisted of tests for the following twelve allergens; Alternaria tenuis, Aspergillus fumigatus, birch, bluegrass, cat, cocklebur, dog, dust mite, maple, oak, plantain, and ragweed. The cut-off point for negative results (Class 0) was established at $0.35 \mathrm{kU}$ per litre. Medical claims captured data on office visits, immunotherapy treatment, skin testing, and slgE allergy testing. Prescription drug claims included prescribed medication use such as antihistamine eye drops, decongestants, intranasal corticosteroids, and oral antihistamines. Over-the-counter (OTC) allergy medications used were not documented in the medical claims, but OTC use was captured in the self-administered questionnaire developed for this study.

\section{Questionnaire development}

Instrument development began with an extensive search for published and validated instruments from the allergy literature including MEDLINElisted and peer-reviewed studies. Content validity was assessed using an expert panel of 10 individuals, including allergists, family physicians, and educators in the immediate area who were knowledgeable in research methodology. After revision, this group agreed that the content of the preliminary instrument was consistent and inclusive for the study objectives. Face validity was established through pre-testing in two practice sites; 16 patients were recruited from one allergy practice and 29 patients were recruited from a family physician practice site. 
The final instrument covered patient demographics including patient history and patient experience with allergies, and HRQoL. In the first section of the questionnaire, information covering patient's knowledge about their diagnosis was compared to a self-assessment of having allergies. Patients were asked to reveal whether they were currently seeing a family physician, allergist, or using a self-initiated strategy to manage allergy symptoms. Other questions asked about a family history of allergies and the extent to which patients had experienced either skin testing and/or slgE testing for allergic rhinitis, and the impact of allergic rhinitis on their ability to sleep. In addition, responses were elicited concerning the perceived value of testing and actual experience with prescribed antihistamine use, intranasal corticosteroids, and OTC products. Patients were asked to rate overall allergy symptom severity on a scale from none to very severe. Work productivity and patient HRQoL were developed from questions adapted from an existing instrument with well-established reliability and validity. ${ }^{21,22} \mathrm{~A}$ ten-point scale was used to assess the impact of allergy symptoms on work performance adapted from the Allergy-Specific Work Productivity and Activity Impairment (APAl-AS) questionnaire. ${ }^{23}$ Certain demographic variables such as age, ethnicity, and gender were not elicited in the survey in order to maintain patient anonymity. However, gender was obtained from patient medical records. The Institutional Review Boards of the University where the research was conducted, and the Henry Ford Health System, approved this study.

\section{Data analysis}

Differences among family physicians, allergists, and self-managed patients with respect to family history, testing, and perceived value of testing were assessed using the Chi-square test. Analysis of variance (ANOVA) and regression analysis were used to assess the impact of the independent variables on work productivity. Correlations were examined to assess the hypothesised relationship between symptoms and productivity. Assumptions of normality, linearity, and equal variances among the groups were assessed to ensure appropriate interpretation of statistical analyses. Statistical analysis was performed using the Statistical Package for Social Sciences version 12 (SPSS Inc., Chicago, Illinois).

\section{Results}

From the sample of 2,065 members of the enrolment scheme who met the study criteria, there were 15 surveys returned as undeliverable, leaving 2,050 in the sample population. From the 652 received questionnaires, respondents who failed to complete $\geq 85 \%$ of the items were not included in the final analysis. Seventy-five responses were of this nature, yielding 577 usable questionnaires - a response rate of $28.1 \%$. As a check for response bias, early respondents (selected from August 1st to August 15th) were compared with late respondents (selected from September 1st to September 15th) since late respondents are more likely to resemble nonrespondents in survey research. ${ }^{24}$ No statistically significant differences were noted in the pattern of responses for variables presented in Table 1 for the early versus late respondents.

Table 1 presents cross-sectional baseline characteristics for the 577 respondents with respect to the three care groups: 240 (41.6\%) reported receiving care from a family physician; $172(29.8 \%)$ received care from an allergist; and 165 (28.6\%) were self-managed. Information regarding gender was gleaned from prescription claim records and revealed that a majority of respondents were female $(375 / 577,65.0 \%)$. Loss of work productivity during the week did not differ significantly among the three groups. On average, respondents reported one hour of work missed per week because of allergy symptoms (range $=0-32$ hours). Significant differences were noted for symptom severity among the three groups $(p<0.05)$ with family physicians seeing patients with more severe symptoms such as sneezing and runny or itchy nose compared to allergists or those patients selfmanaging their symptoms. A larger proportion of patients with no history of allergies was observed in the 'family physician' and 'self-managed' groups. Results revealed that many self-managed patients were actually tested prior to their inclusion in this study, either by another family physician or allergist (family physicians at HFHS do not usually perform skin testing); however, they continued to self-manage their allergy symptoms. With the exception of having the slgE blood test, significant differences were observed among the groups with respect to the variables under investigation. Results revealed that the number of years with allergies was significantly fewer $(p<0.05)$ for patients who received care from a family physician compared to an allergist. Significant differences between observed and expected frequencies using the Chi-square test were revealed for family history and skin testing for the 'allergists' group compared to the 'family physician' and 'self-managed' patients. A higher proportion of patients underwent evaluation for family history of allergies and 
Table 1 Patient Characteristics*

\begin{tabular}{|c|c|c|c|c|}
\hline & $\begin{array}{c}\text { Family } \\
\text { physician } \\
(n=240)\end{array}$ & $\begin{array}{l}\text { Allergist } \\
(n=172)\end{array}$ & $\begin{array}{c}\text { Self- } \\
\text { manage } \\
(n=165)\end{array}$ & $\underset{\text { value }^{* *}}{\mathrm{P}}$ \\
\hline \multicolumn{5}{|l|}{ Informed of allergies by doctor, no (\%) } \\
\hline Yes & $213(89.1)$ & $170(98.8)$ & $129(78.2)$ & \\
\hline No & $17(7.1)$ & $2(1.2)$ & $28(17.2)$ & \\
\hline Don't know & $9(3.8)$ & 0 & $6(3.7)$ & \\
\hline \multicolumn{5}{|l|}{ Think you have allergies, no. (\%) } \\
\hline Yes & $219(91.3)$ & $170(99.4)$ & $139(84.8)$ & \\
\hline No & 0 & 0 & $1(0.6)$ & \\
\hline Don't know & $21(8.8)$ & $1(0.6)$ & $24(14.6)$ & \\
\hline Years with allergies, mean (SD) & $20.3(14.7)$ & $25.3(13.1)$ & $22.8(15.5)$ & 0.001 \\
\hline Health (quality of life), mean (SD) ${ }^{a}$ & $2.88(1.2)$ & $2.61(1.0)$ & $2.79(1.1)$ & \\
\hline Work productivity impact, mean (SD) ${ }^{\mathrm{b}}$ & $1.98(2.6)$ & $1.86(2.4)$ & $1.72(2.4)$ & NS \\
\hline \multicolumn{5}{|l|}{ Allergy symptom severity, mean $(S D)^{c}$} \\
\hline Sneezing & $2.4(0.98)$ & $2.2(0.88)$ & $2.1(0.88)$ & 0.007 \\
\hline Runny nose & $2.4(1.1)$ & $2.2(0.98)$ & $2.1(1.0)$ & 0.044 \\
\hline Itchy nose & $2.5(1.1)$ & $2.3(1.1)$ & $2.2(1.1)$ & 0.039 \\
\hline Watery eyes & $2.5(1.2)$ & $2.5(1.1)$ & $2.5(1.0)$ & NS \\
\hline Nasal congestion & $3.0(1.1)$ & $2.8(1.1)$ & $2.7(1.1)$ & NS \\
\hline Family history of allergies, no (\%) & & & & 0.004 \\
\hline None & $90(50.6)$ & $50(34.7)$ & $63(52.5)$ & \\
\hline Mother & $42(23.6)$ & $40(27.8)$ & $28(23.3)$ & \\
\hline Father & $25(14.0)$ & $32(22.2)$ & $16(13.3)$ & \\
\hline Both & $21(11.8)$ & $22(15.3)$ & $13(10.8)$ & \\
\hline \multicolumn{5}{|l|}{ Diagnostic testing performed for allergies } \\
\hline Skin test & & & & 0.001 \\
\hline Yes & $147(62.8)$ & $162(94.2)$ & $96(60.8)$ & \\
\hline No & $87(37.2)$ & $9(5.3)$ & $62(39.2)$ & \\
\hline Specific IgE blood test & & & & 0.143 \\
\hline Yes & $39(20)$ & $39(28.5)$ & $38(27.3)$ & \\
\hline No & $156(80)$ & $98(71.5)$ & $101(72.7)$ & \\
\hline Testing (blood/skin) was valuable & & & & 0.011 \\
\hline Yes & $66(55.0)$ & $82(73.9)$ & $56(65.1)$ & \\
\hline No & $54(45)$ & $29(26.1)$ & $30(34.9)$ & \\
\hline \multicolumn{5}{|l|}{ Medication use, mean (SD) ${ }^{d}$} \\
\hline Prescribed antihistamines & $3.4(1.7)$ & $3.7(1.7)$ & $2.1(1.6)$ & $<0.001$ \\
\hline Intranasal corticosteroids & $2.4(1.7)$ & $3.0(1.8)$ & $1.8(1.4)$ & $<0.001$ \\
\hline Over-the-counter medications & $1.9(1.4)$ & $1.7(1.3)$ & $1.9(1.4)$ & NS \\
\hline \multicolumn{5}{|c|}{$\begin{array}{l}\text { * Data based on responses for each item - total number of responses do not in every case equal the total number of } \\
\text { questionnaires received because of missing information for some responses }\end{array}$} \\
\hline \multirow{2}{*}{\multicolumn{5}{|c|}{$\begin{array}{l}\text { ** ANOVA and Chi-square test results - two categories used for history (none / yes) } \\
\text { a Scale: } 1=\text { excellent, } 6 \text { = very poor }\end{array}$}} \\
\hline & & & & \\
\hline \multicolumn{5}{|c|}{ b Scale: $1=$ no effect on work, $10=$ prevented work } \\
\hline \multicolumn{5}{|c|}{ c Scale: $1=$ none, $5=$ very severe } \\
\hline d Scale: $1=$ none, $5=4$ weeks or more us & & & & \\
\hline
\end{tabular}


Table 2 Regression analysis of variables influencing work productivity for patients with allergic rhinitis*

\begin{tabular}{lccc}
\hline Variables & $\begin{array}{c}\text { Standardised } \\
\text { coefficients } \\
\text { (beta) }\end{array}$ & t-value & p value \\
\hline Sleep $^{\mathrm{a}}$ & 0.269 & 5.67 & $<0.001$ \\
HRQoL $^{\mathrm{b}}$ & 0.223 & 5.38 & $<0.001$ \\
Watery eyes & 0.14 & 2.96 & 0.003 \\
Sneezing & 0.13 & 2.87 & 0.004 \\
Prescribed antihistamines & & \\
Nasal congestion & 0.108 & 2.71 & 0.007 \\
* $\mathrm{F}=44.51$ p<0.001; $\mathrm{R}$ square $=0.454, \mathrm{n}=438$ & 0.056 & 1.12 & 0.261 \\
a Scale: $1=$ not troubled, $7=$ extremely troubled & & & \\
b Scale: $1=$ excellent, $6=$ very poor & & & \\
c Scale: Weeks of use & & &
\end{tabular}

skin testing by allergists. The value of testing as perceived by patients differed significantly $(p<0.05)$ with respect to care groups. For patients seeing family physicians and who had been tested, $66(55.0 \%)$ reported that the test (blood/skin) was valuable, as compared to 82 patients $(73.9 \%)$ seeing allergists and $56(65.1 \%)$ of the self-managed patients who had previously been tested at one time or another. The use of prescribed antihistamines and intranasal corticosteroids was significantly higher for patients seen by allergists.

A multiple linear regression analysis was performed to examine the effect of the independent variables on work productivity (Table 2 ). The overall $F$ was significant $(p<0.001)$ indicating that some of the variables contributed to the significance of the regression model. The standardised coefficients indicate the weight or effect of each independent variable on the dependent variable controlling for other variables in the model. With the exception of nasal congestion, all variables in the final regression model using the method ENTER were significant $(p<0.05)$ and $45.4 \%$ of the variance was explained in the dependent variable consisting of work productivity. The ability to sleep, and overall HRQoL, had the greatest effect on work productivity, followed by watery eyes, sneezing, and prescribed antihistamine use.

\section{Discussion}

According to the findings of this study, patients seen by family physicians, allergists, and those who self-managed their allergy symptoms, differed with respect to years having allergies, family history, diagnostic testing for allergies, immunotherapy, perceived value of skin or blood testing, and use of prescribed medications. Skin testing was the primary mode for diagnostic evaluation used by allergists, although trends for slgE blood testing were similar yet not as pronounced because of limited opportunities for testing by family physicians at the time the study was implemented. Nonetheless, patients perceived that both tests were valuable. Also noteworthy is the finding that current use of immunotherapy was higher among patients seen by allergists.

Results from the regression analysis demonstrate how sleep, HRQoL, symptoms, and prescribed medication use are associated with work productivity. Consistent with findings from other studies, ${ }^{25}$ an important consequence of allergic rhinitis was its ability to cause poor sleep, which had the greatest impact on work productivity. Although elicited responses for HRQoL were in general 'good' for this population, the correlation was positive indicating that worsening levels of HRQoL had an adverse effect on work productivity. The finding was also apparent for symptoms, especially the observation that watery eyes and runny nose (nasal congestion was not significant) were likely to have an adverse effect on work productivity. Symptom duration was not a significant predictor in the regression model. The greater the impact of allergic rhinitis on work productivity the more likely patients were to take prescribed antihistamines. These results are interesting given the recent concerns about health in the work place, ${ }^{26}$ the impact on health care benefit decisions, and the potential benefits from newer prescribed antihistamines. ${ }^{27}$ 
According to the American Academy of Allergy, Asthma, and Immunology (AAAAI), specific allergen avoidance and treatment measures should be based on positive history and diagnostic testing. ${ }^{28}$

Although it is plausible that patients will find acceptable solutions to cope with allergy symptoms over a longer time frame, patients selected in this study were recently diagnosed with allergic rhinitis. The observation that symptoms appear to be less severe for patients seeing allergists compared to those patients who visited family physicians is likely to be a result of the healthcare process. In a managed care environment, certain criteria specified in clinical protocols (e.g., avoidance, initial trial of medication) are most likely to be satisfied before family physicians refer patients to allergists or other specialists. Allergists would have the opportunity to initiate immunotherapy after further evaluation of patient history, test results, and consideration of disease severity. Thus, for patients who undergo testing and receive appropriate treatment, they may experience a more accurate diagnosis ${ }^{29-31}$ and better symptom control, which may be linked to the perceived value of the test. For self-managed patients who had less severe symptoms, an examination of patient visits revealed that these patients had fewer office visits, and therefore OTC medications may be sufficient for symptom control.

Emphasis on classification of allergic rhinitis by symptom length and symptom severity is important. ${ }^{32}$ However, correct classification per se is no panacea for predicting the impact of allergic rhinitis on work productivity. Appropriate clinical evaluation and an accurate diagnosis using slgE testing and/or skin testing is needed to develop a holistic approach for treatment. ${ }^{33,34}$ From a managed care perspective in the United States, family physicians play a pivotal role in allergy diagnosis: firstly, they have the opportunity to identify patients who would be likely to benefit from an slgE test, which can be performed during a typical office visit; secondly, they refer patients with persistent and more severe symptoms to allergists for further evaluation and treatment; and thirdly, they manage patients with mild symptoms at a minimal cost, which may include the use of OTC medications. However, some managed care organisations have standard protocols and procedures for handling patients suspected of having allergic rhinitis. Results from this study suggest that confirmation of allergic disease may be more complex, perhaps involving a concerted effort by health professionals, which may be more feasible in some health care systems compared to others. Primary care physicians might perform initial evaluation, testing, and treatment involving the short-term use of antihistamines to evaluate response to drug therapy, and patients with more severe symptoms may require extensive evaluation and referral.

Although a direct link between testing and work productivity was not revealed, patients reported that testing was valuable and quantitative test results obtained early in the process should help family physicians provide more efficient and appropriate care to patients that emphasises HRQoL. ${ }^{35}$ Given the results of this study, current guidelines should be reconsidered and possibly changed to enable family physicians to perform slgE testing. Finally, when resources are used efficiently, appropriate patient referrals will provide more information to allergists, especially if these patients have had slgE blood testing before their re-evaluation.

Some of the limitations of this study include the use of prescription and medical claims databases, which are subject to errors such as missing data, inaccurate information, and may not provide a comprehensive account of patient visits and outpatient consultations. ${ }^{36}$

The most important limitation to address is the low response rate. Previous recommendations support as many as four strategies to determine the effect of potential non-response bias on study results. ${ }^{37}$ In this study, the response rate, response variables, and respondents were examined for their potential impact on response bias. Firstly, in this study, the response rate in terms of age, gender, the numbers of patients with or without allergy symptoms, and their symptom severity, is consistent with findings from other studies in this area that use large samples from managed care organisations. ${ }^{38}$ Secondly, exposure prevalence, where patient recruitment could be influenced by the timing of study participation: ${ }^{39}$ in this study, the proportion of respondents in each of the study groups ('family physician', 'allergist', 'selfmanaged') is consistent with the patterns that would be expected under a managed health care system in the United States. That is, along with other conditions such as asthma, the general approach for early detection and diagnosis of allergic rhinitis is largely the work of health professionals in primary care. ${ }^{40}$ Thirdly, work productivity was the dependent variable for the study. A cross-classification analysis was performed for the wave of early, middle, and late respondents to determine if a differential response effect was revealed for work productivity: the results obtained from this 
analysis were non-significant, indicating a consistent response pattern for the dependent variable. Fourthly, although early respondents were compared with late respondents by analysing the variables under investigation for this study, it is possible that response bias may be reinforced by recruitment strategies that allow only the inclusion of early respondents. ${ }^{41}$ Nevertheless, the proportion of patients in this study with mild symptoms was dispersed equally with respect to the response rate from each mailing. Thus, our findings suggest that patients with milder symptoms of allergic rhinitis are likely to manage their symptoms with OTC medications whilst patients with more severe symptoms seek formal evaluation from either primary care physicians or allergists. These findings are consistent with a step care approach in managed care, thus mitigating the possibilities of recruitment bias for example, only enrolling patients with severe cases of allergic rhinitis or patients with a possible misdiagnosis of allergic rhinitis. According to our inclusion criteria, patients selected were recently diagnosed with allergic rhinitis. Although there is no evidence from previous studies to suggest that differences in gender would influence outcomes or that patient quality of life would differ for similar patients who were enrolled in HAP over a longer time frame, future research is needed to address potential biases involving an interaction between patient selection and maturation. ${ }^{42}$ Finally, the extent to which patients are influenced by allergic rhinitis may be attributed to seasonal fluctuations of this geographic region (Midwestern United States): however, seasonal effects or the season in which the test is performed may be of no consequence in this study since the slgE test is unaffected by antihistamine use and the presence of IgE is not affected by the season in which the test is performed.

\section{Conclusion}

The challenges of diagnosing and evaluating allergic disease continue. In addition to the initial assessment of patients suspected of having allergic rhinitis, this study provides evidence that HRQoL, prescribed medication use, and symptom severity are related to work productivity. However, differences with respect to the three care groups may in part be attributed to a care structure that promotes access to primary care physicians prior to specialist referral, which is characteristic of the managed care environment in the United States. Results from this study would support the use of
slgE testing by family physicians as an easy and practical approach to identify patients suspected of having allergic rhinitis. Managed care administrators may want to reconsider current protocols to allow a broader scope of practice by family physicians, which includes the use of slgE testing. Patients with very severe symptoms may require referral. In addition to diagnostic evaluation, other therapeutic options may produce optimal outcomes for patients with very mild symptoms. In terms of resources, HRQoL considerations, changing economic markets, and escalating costs associated with the advent of more expensive allergy treatment options (e.g., leukotriene receptor antagonists, anti IgE treatment), ensuring the appropriate diagnosis is paramount for both patients and care providers in any environment. This study suggests that patient care is optimal if allergists and family physicians have access to appropriate diagnostics such as skin testing and serological tests for slgE antibodies. When used in conjunction with a good family history and clinical evaluation, diagnostic testing followed by appropriate treatment may improve work productivity.

\section{Acknowledgements}

This research was supported from an unrestricted educational grant from Phadia US Inc., formerly Pharmacia Diagnostics.

\section{Conflict of interest declaration}

There are no conflicts of interest to declare.

\section{References}

1. Malone DC, Lawson KA, Smith DH, Arrighi HM, Battista C. A cost of illness study of allergic rhinitis in the United States. J Allergy Clin Immunol 1997;99:22-7.

2. Dykewicz MS, Fineman S, Skoner DP, et al. Diagnosis and management of rhinitis: complete guidelines of the Joint Task Force on Practice Parameters in Allergy, Asthma and Immunology. American Academy of Allergy, Asthma, and Immunology. Ann Allergy Asthma Immunol 1998;81(5 Pt 2):478-518.

3. National Institute for Health Care Management. Prescription drug expenditures in 2000: the upward trend continues. Washington, DC: NIHCM; 2001:1-24.

4. Nathan RA, Meltzer EO, Selner JC, Storms W. Prevalence of allergic rhinitis in the United States. J Allergy Clin Immunol 1997;99:S808-14.

5. Sly R. Changing prevalence of allergic rhinitis and asthma. Ann Allergy Asthma Immunol 1999;82:233-52.

6. Settipane RJ, Hagy GW, Settipane GA. Long-term risk 
factors for developing asthma and allergic rhinitis: a 23year follow-up study of college students. Allergy Proc 1994;15:21-5.

7. Selner JC, Sullivan TJ, Ahlstedt S et al. Current issues relating to in vitro testing for allergen-specific IgE: a workshop report. Ann Allergy Asthma Immunol 1999;82:407-12.

8. Emanuel IA. In vitro testing for allergy diagnosiscomparison of methods in common use. Otolaryngolog Clin North Am 1998;31:27-33.

9. Patorello EA, Icorvaia C, Ortolani C et al. Studies on the relationship between the level of specific IgE antibodies and the clinical expression of allergy: 1. Definition of levels distinguishing patients with symptomatic from patients with asymptomatic allergy to common aeroallergens. J Allergy Clin Immunol 1995;96:580-7.

10. Williams PB, Barnes JH, Szeinbach SL, Sullivan TJ. Analytic precision and accuracy of commercial immunoassay for specific IgE: establishing a standard. J Allergy Clin Immunol 2000;105:1221-30.

11. Soderstrom L, Kober A, Ahlstedt $\mathrm{S}$ et al. A further evaluation of the clinical use of specific IgE antibody testing in allergic disease. Allergy 2003;58:921-8.

12. Cuffel B, Wamboldt M, Borish L, Kennedy S, Crystal-Peters J. Economic consequences of comorbid depression, anxiety, and allergic rhinitis. Psychosomatics 1999;40(6):491-6.

13. Bell IR, Jasnoski ML, Kagan J, King DS. Depression and allergies: survey of a non-clinical population. Psychother Psychosom 1991;55:24-31.

14. Scadding GK, Richards DH, Price MJ. Patient and physician perspectives on the impact and management of perennial and seasonal allergic rhinitis. Clin Otolaryngol 2000; 25:551-7.

15. Blanc PD, Trupin L, Eisner M, et al. The work impact of asthma and rhinitis: findings from a population-based survey. J Clin Epidemiol 2001;54:610-18.

16. Crystal-Peters J, Crown WH, Goetzel RZ, Schutt DC. The cost of productivity losses associated with allergic rhinitis. Am J of Manag Care 2000;6:373-8.

17. Juniper EF. Quality of life in allergic rhinitis. Ann Allergy Asthma Immunol 1999;83:449-54.

18. Meltzer EO, Nathan RA, Selnar JC, Storms W. Quality of life and rhinitis symptoms: results of a national survey with the SF-36 and RQLQ questionnaires. J Allergy Clin Immunol 1997;99:S815-19.

19. DeGraafin't Veld T, Koenders S, Garrelds IM, Vanwijk RG. The relationships between nasal hyperreactivity, quality of life, and nasal symptoms in patients with perennial allergic rhinitis. J Allergy Clin Immunol 1996;98:508-13.

20. Meltzer EO, Casale TB, Nathan RA, Thompson AK. Oncedaily Fexofenadine HCL improves quality of life and reduces work and activity impairment in patients with seasonal allergic rhinitis. Ann Allergy Asthma Immunol 1999;83:311-17.

21. Reily MC, Tanner LA, Meltzer EO. Allergy impairment questionnaires: validation studies. J Allergy Clin Immunol 1996; 97:989.

22. Ware JE, Kosinski M, Dewey JE, Gandek B. How to score and interpret single item health status measures: a manual for users of the SF-8 health survey. Lincoln, RI: Quality Metric Incorporated, 2001.

23. Reilly MC, Zbrozek AS, Dukes EM. The validity and reproducibility of a work productivity and activity impairment instrument. PharmacoEconomics 1993;4(5):353-65.

24. Overton TS, Armstrong JS. Estimating non-response in mail surveys. J Mark Res 1977; 14:396-402.

25. Leger D, Annesi-Maesano I, Carat F et al. Allergic rhinitis and its consequences on quality of sleep: An unexplored area. Arch Int Med 2006;166(16):1744-8.

26. Greenberg P, Birnbaum HG, Kessler RC, Morgan M, Stang P. Impact of illness and its treatment on workplace costs: regulatory and measurement issues. $J$ Occup Environ Med 2001;43:56-63.

27. Okubo K, Gotoh M, Shimada K, Ritsu M, Okuda M, Crawford C. Fexofenadine improves the quality of life and work productivity in Japanese patients with seasonal allergic rhinitis during peak cedar pollinosis season. Int Arch Allergy Immunol 2005;136:148-54.

28. American Academy of Allergy, Asthma, and Immunology. The Allergy Report. Milwaukee, WI: AAAAl; 2001.

29. Williams PB, Siegel C, Portnoy J. Efficacy of a single diagnostic test for sensitization to common inhalant allergens. Ann Allergy Asthma Immunol 2001;86:196-202.

30. Szeinbach SL, Williams PB, Muntendam P, O'Connor RD. Identification of allergic disease among users of antihistamines. J Manag Care Pharm 2004;10(3):234-8.

31. Gendo K, Larson EB. Evidence-based diagnostic strategies for evaluating suspected allergic rhinitis. Ann Intl Med 2004;140(4):278-89.

32. Hoecke VH, Vastesaeger $N$, Dewulf L, Sys L, van Cauwenberge P. Classification and management of allergic rhinitis patients in general practice during pollen season. Allergy 2006;61:705-11.

33. Douglass JA, O'Hehir RE. Diagnosis, treatment and prevention of allergic disease: the basics. Med J Australia August 2006;185(4):228-33.

34. Nolte F, Nepper-Christensen S, Backer V. Unawareness and undertreatment of asthma and allergic rhinitis in a general population. Respir Med 2006;100(2):354-62.

35. Majani G, Bairardini I, Giardini A et al. Health-related quality of life assessment in young adults with seasonal allergic rhinitis. Allergy 2001;56:313-17.

36. Anandan C, Simpson CR, Fischbacher C, Sheikh A. Exploiting the potential of routine data to better understand the disease burden posed by allergic disorders. Clin Experiment Allergy 2006;36:866-71.

37. Stang A. Nonresponse research - an underdeveloped field in epidemiology. Eur J Epidemiol 2003;18:929-31.

38. Bousquet J, Neukirch F, Bousquet PJ, Gehano P, Klossek JM, Le Gal M, Allaf B. Severity and impairment of allergic rhinitis in patients consulting in primary care. J Allergy Clin Immunol 2006;117:158-62.

39. Stang A, Jockel KH. Studies with low response pro-portions may be less biased than studies with high response proportions. Am J Epidemiol 2004;159(2):204-10.

40. Levy ML. Occupational asthma case finding: a role for primary care. Brit J Gen Pract 2004;54(507):731-3.

41. Holle R, Hochadel M, Reitmeir P, Meisinger C, Wichmann H. -Erich. Prolonged recruitment efforts in health surveys: effects on response, costs, and potential bias. Epidemiology 2006;17(6):639-43.

42. Campbell DT, Stanley JC. Experimental and quasiexperimental designs for research. Chicago: Rand McNally, 1963.

Available online at http://www.thepcrj.org 